\title{
LIRE LES FAITS DE STRUCTURE DU LYS \\ DANS LA VALLEE: INTROSPECTION, RÉTROSPECTION ET RÉTROACTION*
}

\section{Laure Lévêque**}

Résumé: C'est peu de dire que Le Lys dans la vallée de Balzac jouit d'un statut de "texte sacré », à tel point qu'il en étouffe sous un glacis critique qui le fige. Le présent article voudrait redonner la parole à un texte que Balzac concevait comme un pivot - esthétique, éthique, sémantique - de son grand auvre. Cela imposait de le passer au crible d'une approche plurielle-génétique, générique, narratologique, métatextuelle aussi, intégrant l'inscription, maintes fois repensée, du Lys dans l'économie générale de la Comédie humaine. Or; à tous ces niveaux, c'est une poétique de l'ambiguitté qui prévaut, dans cette écriture en double registre où, véritable Pénélope, l'auteur défait l'cuvre du narrateur, et, vice versa. Chez Balzac, le Penseur et l'Écrivain sont deux instances qui se contrarient. Derrière les atermoiements, les refontes et les repentirs qui accompagnent l'histoire éditoriale du texte, la figure de l'intellectuel critique se fait voir par le moyen d'une écriture qui se dénie elle-même.

Le Lys occupe une place à part dans la production balzacienne. D'une part parce qu'il circule à l'intérieur des cloisonnements qu'établit la Comédie Humaine: originellement prévu pour les "Scènes de la vie de campagne", l'inachèvement du Député de province contraint Balzac à déplacer le Lys vers les "Scènes de la vie de province". Et ce n'est que sur indication corrigée de l'édition Furne', qu'après un exil de trois ans, le Lys réintègre le milieu

\footnotetext{
* Recebido para publicação em 17 de março de 2006.

** Université de Franche-Comté.

' Furne, Dubochet et cie., Hetzel et Paulin, sont les éditeurs de Balzac, indiqués de façon conventionnelle par le terme «Furne»; c'est la seule édition des œuvres complètes de Balzac, entièrement contrôlée par l'auteur et lue par ses contemporains. Les dates de parution des volumes se situent entre juin 1842 et novembre 1848 .
} 
rural où, aujourd'hui encore, il fleurit et avec lequel la critique l'a si souvent confondu.

En même temps qu'il a évidemment tout d'une scène de la vie privée.

De plus, on ne sait exactement quel statut générique lui accorder. Roman épistolaire? Mais Balzac en a commis un, et ce sont les Mémoires de deux jeunes mariées, qu'on est effectivement bien en peine de lui comparer. Du reste, Jean Rousset ne s'y trompe pas et lui dénie cette qualité, au prix d'ailleurs d'acrobaties génériques puisqu'il propose de forger le concept hasardeux de "roman autobiographique au passé" (ROUSSET,1962:100). Mais ce statut, déjà éminemment flottant en lui-même, est encore inquiété par la concurrence interprétative de la critique quand Alice Mura y revient et pose ouvertement la question: "le Lys, un roman épistolaire?", pour finalement.trancher en faveur du "roman par lettres" (MURA, 1993:44-45)2. Heinz Weinman, de son côté, compliquant encore cette combinatoire touffue, conclut au "roman autobiographique par lettres" (WEINMAN, 1975:21-41).

Une interrogation sur les modèles génériques structurants du $L y s^{3}$ conduirait au même brouillage: histoire d'une passion, roman d'apprentissage -ou d'initiation -, roman courtois, roman mystique... Sans cesse, Le Lys brasse des modèles repérables, connus, mais dont l'agencement pose problème et vient gêner la conclusion, comme si Le Lys s'employait à n'être jamais que du côté de l'envers des histoires contemporaines.

Serait-il possible de sémantiser cette résistance du texte et de montrer, à travers l'analyse des structures du roman - de celles qui organisent le sens et des structures profondes qui ouvrent sur le mythe -, comment, derrière une écriture qui se revendique de la transparence, tout un réseau de signaux d'alerte se déploie pour inviter à cultiver un autre Lys dans une relecture qui a aussi valeur de réaction?

La forme épistolaire - qu'il faut bien se résoudre à interroger dans la mesure où, dans leurs désaccords mêmes, tous les balzaciens la reconnaissent au moins pour dominante à l'intérieur du dispositif textuel — n'est pas première dans Le Lys. À l'origine, la structure romanesque obéissait à une manière

2 Par lettre serait d'ailleurs infiniment plus juste, paradoxe à part.

${ }^{3}$ Sauf mention contraire, toutes les références faites au Lys dans la vallée renvoient à l'édition qu'en a donnée Jean-Hervé Donnard dans la Pléiade, t.IX, 1978. En outre, le titre du roman sera ici souvent indiqué simplement par Le Lys ou par Lys. 
balzacienne éprouvée: le récit enchâssé4, qui s'ouvrait sur la célébrissime scène du bal. La maturation vers l'épistolarité est lente: au corps du récit s'agrègent successivement: l'envoi à Natalie, qui date du premier dossier d'épreuves (Revue de Paris, novembre 1835); la réponse de Natalie, plus tardive encore (probablement de juin 1836); les apostrophes à la destinataire, corollaires obligés, qui se rajoutent à partir des épreuves de l'édition préoriginale dans le but évident de renforcer l'hypothèse épistolaire.

La réversion du récit personnel vers le cadre épistolaire peut paraître au moins curieuse dans un roman où le tabou du non-dit amoureux pèse de tout son poids, mais elle trouve son efficace stylistique en ce qu'elle est formesens de l'émotion intime et rétrospective. Sur ce point, on ne peut que suivre la conclusion de Gabrielle Malandain qui voit "l'énoncé et l'énonciation signifier ensemble" (MALANDAIN,1988:31).

Et, de fait, c'est bien ce que disait la date du 8 août 1827 qui signait l'envoi à Natalie jusqu'à l'édition Charpentier de 1839, date qui correspond au dénouement du Contrat de mariage, où la fuite de Paul de Manerville, ruiné, parti reconquérir une fortune volage aux colonies, réintègre Natalie dans le marché amoureux. Si, dans l'envoi, Félix accepte de "livrer son passé" en "cédant au désir" de sa maîtresse, s'il peut lui lancer un "à ce soir" sans ambiguilté, c'est bien qu'il a tout lieu d'espérer quelque bonne fortune de sa performance narrative et, donc, que le passé gouverne et détermine son $\grave{a}$ venir.

À l'intérieur de la lettre-cadre, outre les lettres répondant à une fonction purement diégétique (recommandation de Félix à la duchesse de BlamontChauvry, au duc de Lenoncourt...), quatre d'entre elles sont reproduites dans l'énoncé. Il s'agit: 1) du commencement d'une lettre de Félix à Madame de Mortsauf, rédigée lors de son premier séjour à Clochegourde (BALZAC, Le Lys:1074-1076); 2) du bréviaire mondain qu'Henriette adresse à Félix avant son entrée dans le monde (Ibidem:1084-1097); 3) d'une lettre de Madame de Mortsauf à Félix, annonciatrice de funestes nouvelles concernant Jacques (Ibidem:1140-1141); 4) de la lettre-confession en forme de testament d'Henriette (Ibidem:1145-1142).

Lesquelles lettres entrent deux à deux dans un système d'opposition

${ }^{4}$ Lequel peut certes se targuer de glorieux ancêtres, entre Gobseck,Honorine, Autre étude de femme, Un Prince de la bohème... 
régi par une problématique de la parole. Parole qui se refuse à s'incarner dans le verbe (lequel, on le sait, est toujours porté à se faire chair) pour les deux premières, qui correspondent, dans l'ordre de la diégèse, au temps suspendu dans un monde encore clos du premier séjour à Clochegourde. Parole dégradée, mondaine, où le signe s'est disjoint de la chose à laquelle il référait, parole sociale certes plus vraie, mais mortifère pour l'individu pour les deux dernières. Le passage suivant, emblématique du Félix parisianisé, livre l'a. b.c. de cette parole frauduleuse :

"Souvent lady Dudley, comme beaucoup de femmes, profitait de l'exaltation à laquelle conduit l'excès de bonheur, pour me lier par des serments; et sous le coup d'un désir, elle m'arrachait des blasphèmes contre l'ange de Clochegourde. Une fois traitre, je devins fourbe. Je continuai d'écrire à Madame de Mortsauf comme si j'étais toujours le méchant enfant en habit bleu qu'elle aimait tant" (Ibidem:1148).

Ainsi se dessine très clairement une opposition marquée entre la première partie du roman, relation du premier séjour à Clochegourde, et une seconde partie d'égale importance, qui ne cesse de tenter de ressaisir ce temps heureux qui n'est plus, ce chronotope évanoui. Disposition antagonique qui emporte avec elle des déterminations multiples: diégétiques, idéologiques il y a recouvrement de l'affrontement Clochegourde/Paris sous cette césure - et stylistiques - quand, à l'exaltation lyrique qui célèbre la complétude, succède le chant élégiaque de la perte et de la déploration. Le decrescendo est manifeste dans la lettre-confession de Madame de Mortsauf qui radicalise encore les conditions de réception de son discours par rapport à sa lettre d'initiation que, déjà, Félix ne devait lire qu'après l'avoir quittée. Henriette y explicite tout ce qui était biaisé, oblique, déguisé, au temps de la parole pré-verbale de l'édénique premier séjour, montrant assez par là combien est fondamentale pour ce texte la question du signe - par nature double, comme on le sait - et de son interprétation. Motivée dans le récit par la mort de l'héroïne, ce véritable mémoire d'outre-tombe relance la lecture, invite le lecteur à re-lire le récit et installe la duplicité au cœur du texte : la "sainte 
de Clochegourde" appartenait, certes au ciel, mais aussi à la terre, et si ses premiers conseils mettaient au-dessus de tout la loi sociale en vertu de la très bonaldienne théorie des devoirs, sa lettre posthume dévoile l'envers de ce code sacrificiel.

Par là, on peut la rapprocher de l'autre texte liminaire: la réponse de Natalie qui clôt le roman, aggrave encore l'éclairage décapant qu'avait jeté Henriette, et sert d'embrayeur à une lecture rétroactive. Et, de fait, Natalie, en qui, on le sait, Victor Brombert (1998:23-36) a repéré le lecteur caché de Balzac, est bien ce critique ultime, maître du métalangage à qui appartient l'analyse dernière du texte.

Un tel dispositif énonciatif, qui accueille les béances de la double stratégie d'écriture - et de la double stratégie de lecture - ainsi induites, ne peut laisser sauf le statut du destinataire. Il est incontestable qu'une lecture littérale du geste d'envoi de Félix à Natalie, conforté par les onze formules d'adresse qu'il glisse dans son texte, semblerait devoir interdire que l'on puisse disputer ce poste à cette dernière. Pourtant, peut-on raisonnablement identifier d'autorité Natalie - cette même Natalie en qui un impeccable décrypteur du social, de Marsay" - reconnaît dans la "froideur naturelle" l'indice le plus sûr d'une "nature siliceuse", le signe irréfragable qu'elle se range parmi les "organisations de fer" qui se distinguent par leur "peu de cœur"6 _ avec ce "vous" par lequel Félix (BALZAC, Le Lys:1139) apostrophe une communauté qui tient des happy few du cœur: "ô vous qui aimez"? (Ibidem)

Quand l'intratextualité balzacienne n'y suffirait pas, le pluriel devrait interdire cette lecture

\footnotetext{
${ }^{5}$ Comme le personnage de Natalie, celui d' Henri de Marsay fait partie du Contrat du mariage, de Balzac.

'Dans la physiologie du mariage que de Marsay adresse au mari malheureux, il est difficile de ne pas reconnaître en Natalie l'archétype de la femme sans cour balzacienne : «Ta femme, cher ami, n'était-elle pas, dans la plus large acception du mot, une femme à la mode ? Elle ne pensait qu'à ses succès, à sa toilette ; elle allait aux Bouffons, à l'Opéra, au bal ; se levait tard, se promenait au bois; dînait en ville ou donnait elle-même à dîner. Cette vie me semble être pour les femmes.ce qu'est la guerre pour les hommes; le public ne voit que les vainqueurs, il oublie les morts. Si les femmes délicates périssent à ce métier, celles qui résistent doivent avoir des organisations de fer, conséquemment peu de cœur, et des estomacs excellents.(...) Ta femme résistait admirablement à cette vie » (BALZAC, Le contrat de mariage, 1984: 242243).
} 
“.../génies éteints dans les larmes, cœurs méconnus, saintes Clarisse Harlowe ignorées, enfants désavoués, proscrits innocents, vous tous qui êtes entrés dans la vie par les déserts, vous qui partout avez trouvé les visages froids, les cœurs fermés, les oreilles closes, ne vous plaignez jamais. Vous seuls pouvez connaître l'infini de la joie" (Ibidem:1038).

Est-ce vraiment à la femme à la mode que Félix tend ici le miroir du proscrit? Ailleurs encore, la communauté des destinataires s'élargit manifestement jusqu'à inclure tout lecteur du roman, quand Félix anticipe de manière flagrante sur ce que Natalie ne peut savoir encore, sauf à s'être procuré la "lettre" de son amant en librairie: "Elle était, comme vous le savez déjà sans rien savoir encore?, le lys de cette vallée" (Ibidem: 987). De fait, comment Natalie pourrait-elle comprendre cette référence au titre du roman alors que Félix n'en est encore qu'au stade introductif de sa confession et n'a pas encore fait jouer le motif lilial de la métaphore?

Voilà donc Natalie quelque peu bousculée dans ses prérogatives. Mais elle devient franchement menacée si l'on veut bien s'apercevoir qu'elle n'est jamais convoquée que dans la contiguité descriptive des splendeurs inviolées — et donc d'autant plus splendides - de Madame de Mortsauf et des charmes enivrants d'Arabelle. Il est plus que douteux, en effet, qu'elle puisse prêter une oreille favorable à cette douteuse apostrophe: "Malheur à qui n'a pas eu son Henriette! Malheur à qui n'a pas connu quelque lady Dudley! (...) Mais heureux qui peut trouver les deux en une seule; heureux, Natalie, l'homme que vous aimez!" (Ibidem)

Il n'est alors que trop évident que Natalie sert avant tout de point de comparaison entre les deux femmes qui l'ont précédée dans le cœur de Félix et trouve sa cohérence et sa définition textuelles dans la synthèse qu'elle opère entre elles. Mais c'est aussi l'un des procédés qui donne à lire de façon transparente ce qui anime Félix, soit l'obsession désespérée de faire se rejoindre le présent avec le passé, puisqu'il pourrait être donné à Natalie de ressusciter les deux femmes en ranimant leurs deux fantômes. Par ces effets

${ }^{7}$ C'est moi qui souligne 
d'écriture, Natalie, personnage invité sur épreuves, participe d'une entreprise concertée de re-création du passé. Elle est le tenseur qui dynamise un récit qui, sans elle, s'écrirait exclusivement au passé, l'embrayeur des oscillations affolées entre un passé dépassé et un présent menacé - dialectique que l'on retrouve dans le principe avoué de la confession, de la confidence comme dans la scission irrémissible entre les deux parties du roman -, le point de fuite et l'aboutissement d'une dynamique héroïque - celle de Félix — dont la perpétuelle indécision fait vaciller le rapport à l'autre.

Car la logique obvie du récit, qui installe la lettre-récit comme confession arrachée à Félix par sa maîtresse - peut-être sous la menace d'une rupture -, truchement devant lui concilier ses bonnes grâces (au reste, il ne lésine pas sur la captatio benevolentiae) devrait au moins inspirer à celui qui la commande, à l'instar de la princesse de Cadignan, attirant et assujettissant d'Arthez dans ses rets, inventant, au passage, le mentir-vrai avant Aragon, la prudence retorse et l'art subtil de certains détours, or, il n'en est rien. Au reste, et à cet égard encore, le personnage de Natalie remplit l'office de fort opportun révélateur, qui ne s'embarrasse pas de nuances pour signifier à Félix qu'il a manqué son but par la sanction sans appel dont elle paie son long récit: "Vous avez manqué de tact envers moi (...) Quand on a
sur la conscience de pareils crimes, au moins ne faut-
il pas les dire. Je vous ai fait une imprudente demande,
j'étais dans mon rôle de femme, de fille d'Eve, le
vôtre consistait à calculer la portée de votre réponse. Il
fallait me tromper; plus tard, je vous aurais remercié."
(Ibidem:1226-1228)

Et, perfide, elle ajoute, renvoyant Félix à ses chères études : "Il est trop tard maintenant pour commencer vos études..." (Ibidem:1229). Si l'on songe que c'est du même judicieux conseil que l'habile de Marsay, dans Le Contrat de mariage (BALZAC, Le Contrat de mariage,1983:257) avait gratifié un autre prétendant de la cruelle Natalie - et plus légitime même puisqu'il s'agit de son mari, Paul, guère mieux payé de ses hommages et sévèrement tancé par son ami d'un "resteras-tu donc toujours lycéen"-, on peut certes renvoyer dos à dos les personnages du gros Paul et du mince Félix et faire de Félix à 
l'envi une cloche... ou une gourde... Il reste que cette identité structurelle trouve d'autres résonances que celles de la grasse plaisanterie et doit inviter à interroger de façon plus nuancée les mécanismes de la production du sens.

Pour en revenir à la stricte économie interne du Lys, comment ne pas voir une contradiction difficilement réductible entre le but implicite que se propose Félix (conquérir Natalie, on n'oublie pas les implications optimistes de son "à ce soir" initial) et l'arsenal de moyens déployés, qui tient du pavé de l'ours? ${ }^{8}$ Il faut un aveuglement au moins singulier pour s'ouvrir d'un "chère Natalie, le bonheur est absolu, il ne souffre pas de comparaison" (Ibidem:1185) alors que Félix s'ingénie précisément à comparer explicitement, non seulement Henriette à Arabelle mais, implicitement, Natalie à l'une et à l'autre ${ }^{10}$. Une telle stratégie discursive, loin de déboucher sur l'heureuse réconciliation des contraires, suscite le spectre de l'aporie. Celle du personnel romanesque - et Natalie souligne bien qu'elle renonce à réunir en elle "I'eau et le feu". Aporie du récit aussi, qui se dénonce ainsi au moment même où il s'énonce, qui jette la suspicion sur sa propre performance.

Toujours, Félix s'ingénie à nier la Natalie "réelle" au profit d'une Natalie qui syncrétiserait la double figure d'Henriette et d'Arabelle (tâche d'autant plus effrayante que Madame de Mortsauf est elle-même éclatée en Blanche

${ }^{8}$ Natalie aura alors beau jeu de donner cours à son ironie: "N'avez-vous donc jamais compris la vertu des hommes à bonnes fortunes? Ne sentez-vous pas combien ils sont généreux en nous jurant qu'ils n'ont jamais aimé, qu'ils aiment pour la première fois?" (Le Lys:1228). Et, toujours: "Il est trop tard maintenant pour commencer vos études, pour apprendre à nous dire ce que nous aimons à entendre (Ibidem:1229).

${ }^{9}$ On en trouve l'expression la plus achevée dans un dualisme qui fait honneur au cartésianisme de notre héros: "Lady Arabelle était la maîtresse du corps, Madame de Mortsauf était l'épouse de l'âme" (Ibidem:1146)

${ }^{10}$ Mais cet implicite est si grossier que Natalie ne peut s'y leurrer, qui entend bien défendre sa singularité et bâtit son argumentaire sur cette ligne de défense: "Vous m'avez donné à entendre que je ne vous aimais ni comme Henriette, ni comme Arabelle. J'avoue mes imperfections, je les connais; mais pourquoi me les faire si rudement sentir ? Savez-vous pour qui je me suis prise de pitié? pour la quatrième femme que vous aimerez.(...) Je renonce à la gloire laborieuse de vous aimer : il faudrait trop de qualités catholiques ou anglicanes, et je ne me soucie pas de combattre des fantômes.(...) je ne veux pas de rivale ni au-delà nì en deçà de la tombe. (...) Votre programme est inexécutable. Être à la fois Madame de Mortsauf et lady Dudley, mais, mon ami, n'est-ce pas vouloir réunir l'eau et le feu ?" (Ibidem:1226-1228) 
et en Henriette, dont le dédoublement répond à celui de Félix-Amédée), de l'épouse de l'âme et de la maîtresse du corps, de l'eau et du feu, de l'ange et du démon, du Ciel et de l'Enfer, du spiritualisme et du matérialisme... identités inassumables que Natalie est priée de réaliser. Peu pressée de donner les mains à sa propre aliénation, elle ne peut - et cela est très vite patent pour le lecteur - que se crisper sur son identité et opposer à Félix un non possumus blessé et hautain. En fait de troisième voie, Natalie est une impasse, mais, lectrice cachée et lectrice lucide, elle démonte les mécanismes qui construisent le récit, elle qui plaint la quatrième femme qu'aimera Félix. Raymond Mahieu conduit plaisamment cette même analyse, qui stigmatise en Félix l'inadéquation des fins visées et des moyens requis:

"A l'inverse d'un opérateur avisé qui hypothéquerait le capital qu'il a accumulé (son passé) en vue d'acquérir d'autres biens prometteurs d'une plus-value (son avenir), Félix se propose d'engager son avenir pour payer la récupération, par son récit, d'un passé qu'il sait totalement dévalué. On excusera aisément le refus de Natalie d'être partie prenante d'une affaire aussi douteuse, sur laquelle personne, à commencer par celui qui l'échafaude, ne se fait d'ailleurs d'illusion " (MAHIEU, 1993:104)

Cette négation de Natalie s'intègre dans la logique de l'aveu qu'a mise en évidence Lucienne Frappier-Mazur (1976) à propos du Lys, logique supérieure à celle que semblait postuler la diégèse, mais que l'énonciation s'ingénie à combattre. Lorsque Madame de Mortsauf à l'agonie révèle ce qu'un meilleur lecteur que Félix aurait dès longtemps décrypté — "tout a été mensonge dans ma vie" (BALZAC, Le Lys:1201) - les soupirs de la sainte qu'elle exhale, loin de susciter un quelconque assouvissement - fûtil sur le mode du trop tard - ne provoque, chez celui qui les recueille, que "l'épouvante" (Ibidem:1201), et Félix se jette avec gratitude sur l'explication bien commode et pré-naturaliste que lui fournit le père de Dominis: ce sont les fleurs, il est vrai plus que huysmansiennes"

"Et, certes, le passage suivant pourrait à bon droit lui être imputé: "enfin, tout ce que ces naïves créatures ont de plus échevelé, de plus déchiré, des flammes et des. triples dards, 
que calmera l'opium. Mais ces fleurs ne se laissent pas si aisément emporter hors de la chambre ardente, et l'injonction de l'officieux abbé n'en peut mais : elles sont de l'espèce des vivaces intertextuelles - Madame Jules Desmarets en a déjà fait l'amère expérience dans Ferragus ${ }^{12}$. Si bien que ces fleurs au parfum entêtant poursuivent d'autres buts que de simple ornementation, fut-elle enflée jusqu'au morceau de bravoure comme en ces scènes itératives des bouquets qui défrichent d'autres voies au récit : non pas fleurs de rhétorique mais rhétorique des fleurs. Félix, expert en jeux floraux, le dit avec des fleurs, dont l'agencement devient, entre son lys et lui, "une langue à [leur] usage" (Ibid.:1058) dont le maniement leur procure "un contentement semblable à celui de l'esclave qui trompe son maitre", un code herméneutique second capable de couper l'herbe sous le pied au récit dans son fonctionnement canonique - le "maître", c'est lui aussi —, de revigorer son tronc fatigué d'un peu de sève nouvelle.

La greffe, pourtant, ne prendra pas - du moins dans le terreau du Lys et l'éclosion de ce langage, promu comme signification mais dont l'émergence reste du domaine de l'allusion, devra attendre des jours meilleurs. Alors, lorsque Didon redevenue chrétienne dit: "Oubliez ce que j'aurai pu vous dire, pauvre insensée que j'étais" (Ibidem:1057) en reniant ses rêves sans équivoque de chevauchées, de fuite à la Béatrix en pays italien, Félix montrera un lâche soulagement. Mais l'abbé de Dominis aura beau sonner la charge contre les coupables fleurs et les virtualités germinatives qu'elles portent en elles, elles auront fait leur office et la lettre-testament vient accomplir la promotion du refoulé et nier les occultations.

Cette langue commune des amants, imaginée à leur usage, les réunit encore par delà le tombeau dans la contagion des programmes énonciatifs qui organisent leurs confessions respectives, quand Félix s'inspire du "je ne veux pas que vous ignoriez une seule de mes pensées" (Ibidem:1215) d'Henriette pour adresser à Natalie cette pétition de principe liminaire: "peut-

des feuilles lancéolées, déchiquetées, des tiges tourmentées comme les désirs entortillés au fond de l'âme: Du sein de ce prolixe torrent d'amour qui déborde, s'élance un magnifique double pavot rouge accompagné de ses glands prêts à s'ouvrir, déployant les flammèches de son incendie (...) Quelle femme enivrée par la senteur d'Aphrodise ne comprendra ce rouge désir de l'amour qui demande un bonheur refusé." (Ibidem:1057) ${ }^{12}$ Personnage de Balzac in Ferragus, père de Mme Jules Desmarets, rencontrée dans le Lys. 
être vaut-il mieux que tu saches tout" (Ibidem: 969), pacte de la sincérité et de l'exhaustivité qui aura évidemment sur elle - symétrie oblige - le même effet dévastateur que celui qu'avait déclenché en lui la transparence d'Henriette. Il fallait me tromper! Et l'on peut alors, sans se tromper, lire le récit de Félix comme une réponse, à la fois à la sollicitation imprudente de Natalie, et à la lettre posthume d'Henriette, qui devient du même coup la destinataire véritable du récit, elle qui, de son vivant, s'était fait une religion de proscrire toute communication de cet ordre. Spoliée de ce dépôt même, ne restent à Natalie que les piquantes ressources de l'ironie: "aussi dois-je vous prémunir, dans votre intérêt (...) contre le danger de votre mémoire. (...) Ni le cœur ni les sens ne triompheront jamais de vos souvenirs" (Ibidem:1226).

Lectrice cachée encore, lectrice avertie toujours, elle sait bien que Félix achoppe sur ce qui réclamerait à tout le moins un Gautier ou un Nerval, contenter tout ensemble la vivante et la morte, réussir la symbiose harmonieuse du passé et du présent.

De là procèdent un certain nombre de dysfonctionnements flagrants des normes du récit, si l'on en revient aux diverses hypothèses génériques formulées pour rendre compte du roman, qui sollicitent principalement les codes du roman par lettres, du roman autobiographique, du roman-mémoires. Au croisement de ces trois traditions bien attestées, Félix, homme fait de 33 ans, maître des requêtes siégeant au Conseil d'État et maître espion de Sa Majesté, devrait au moins instaurer une relation de relative maitrise énonciative sur le personnage, égaré et naïf, qu'il était à l'époque de ses années de formation. Or, à ce traditionnel double jeu des points de vue, se substitue un travail textuel plus flottant, plus mouvant. On ne peut nier qu'il y sacrifie et il n'est pas rare de rencontrer, sous la plume de Félix, l'enrichissement rétrospectif classique. Ainsi de sa première visite à Clochegourde où son aspect malingre et souffreteux lui concilie, en dépit du contentieux du baiser volé au bal de Tours, un accueil amène de Madame de Mortsauf parce qu'elle y voit le signe que ses enfants, rachitiques eux aussi, ne sont pas condamnés, retrouvailles que Félix commente ainsi: "ce fut, comme je le sus depuis, le second lien qui l'attacha si fortement à moi" (Ibidem:1003). De la même manière, l'effort de lucidité pour décrypter certains passages problématiques est-il moins imputable au jeune homme qu'il fut qu'à l'homme qu'il est devenu, dont l'analyse s'exerce à distance: 
"Enfin, plus tard, j'ai reconnu la cause de ce bonheur plein. À mon âge, aucun intérêt ne me distrayait le cœur, aucune ambition ne troublait le cours de ce sentiment déchaîné comme un torrent et qui faisait onde de tout ce qu'il emportait. Oui, plus tard nous aimons la femme dans une femme, tandis que de la première femme aimée, nous aimons tout" (Ibidem:1049).

Mais c'est loin d'être le régime dominant, et nous en appelons sur ce point à la brillante synthèse de Jacques Roudaut, dans la préface qu'il fait au Lys dans une édition de 1977:

"loin de liquider le passé en le livrant, l'écriture transpose le passé dans le présent, fait échec au travail de deuil. Félix de Vandenesse refuse la succession temporelle, nie le rôle du temps pour établir une simultanéité des durées: c'est ce que disent dans l'ordre de la construction, les retours en arrière, dans celui de la thématique le rêve des amours contemporaines" (ROUDAUT,1977)

Si bien que si le mode d'énonciation privilégié est bien celui du roman personnel tel qu'il s'est constitué en s'émancipant d'une longue lignée de romans-confessions qui peut se prévaloir de récents et pourtant glorieux ancêtres - depuis René, Adolphe, Aloys, et jusqu'à Volupté -, ces modèles sont constamment subvertis par un Félix qui n'en conserve en quelque sorte que la coquille vide, débarrassée de contraintes génériques qu'il délaisse et combat en s'efforçant d'annihiler la distance entre le moi-narrant et le moi-narré par le double truchement de la coagulation des durées passées et présentes et de la confession en forme de plaidoyer. Par quoi l'on se prive nécessairement des satisfactions propres aux modèles du genre qui mettent l'accent sur la maturation hérö̈que et la résolution des conflits, par quoi l'on renonce aux espaces d'une autre vie - dominée et conquise - pour ne creuser, sans relâche, que l'espace infini - mais bouché, indépassable - du manque et de l'imperfection. Alors, si l'on convoque à nouveau G. Malandain (op.cit.) sur le chapitre de l'homogénéité de l'énoncé et de l'énonciation, il est logique qu'à la posture énonciative d'un héros englué dans un passé qu'il ne 
parvient pas à, justement, dépasser, corresponde, dans l'énoncé, l'inflation du paradigme de l'enfermement passionnel.

Or, tel est bien ce qui gouverne Félix et l'amène à occulter tout ce qui viendrait entraver la célébration lyrique du temps où la communication se passait de mots - ou, du moins savait retrouver le canal de la pureté en posant pour préalable sa radicale altérité d'avec les impostures de la langue des maîtres, véhicule de l'idéologie dominante, que ce soit selon les modalités de Félix - le flower power —, ou selon celles d'Henriette qui, patiente Pénélope, défait fil à fil la trame - et les chaînes - du langage commun qu'elle voudrait renouer en autant de fils conducteurs ${ }^{13}$.Mais les Parques s'en sont emparées avant qu'elle ait terminé son ouvrage, déshabitant du même coup le paradis tourangeau dont Félix, fidèle au régime énonciatif par lui défini - et, par nous, plus haut - , ne peut que s'obstiner à refuser de prendre acte qu'il en a été chassé. Et c'est précisément cette dénégation de la chute qui lui rend celle du récit plus dure. G. Malandain est presque aussi sévère que Natalie, qui diagnostique chez Félix "l'inachèvement de l'apprentissage de la parole", dans un système où "l'accès à la parole sert plus à réfuter la réalité qu'à la comprendre" (MALANDAIN,1988:35). Or, on sait les faits têtus et peut donc s'attendre à les voir faire retour.

Écrire cette confession revient alors pour son auteur à tenter de faire perdurer le désir - ce qui, on en conviendra, était peu à même de se concilier aisément avec les objectifs avoués de la stratégie amoureuse, orientés vers la comtesse de Manerville - et d'abord par le biais des fréquentes protestations d'amour à Natalie, priée d'endosser le rôle inconfortable de succédané de la morte, mais surtout en se faisant le desservant et le chantre de la passion amoureuse. C'est ainsi que Félix se reprend sans cesse aux pièges de l'angélisme alors que la confession de Madame de Mortsauf devrait l'avoir définitivement éclairé sur l'épineuse question du sexe des anges, ce en quoi il n'est pas aussi inconséquent qu'il y parait, c'est-à-dire qu'il suit à la lettre un programme énonciatif qui commande des efforts désespérés pour annuler et le temps et la mort du désir et qui l'enferme nécessairement dans l'inaccompli,

\footnotetext{
${ }^{13}$ De fait, structurellement, les ouvrages de tapisserie de Madame de Mortsauf sont bien donnés, et de son propre aveu même, comme rigoureusement équivalents aux ambitions linguistiques et, on l'a vu, démiurgiques, contenues dans les bouquets de Félix, à qui elle confie: "Ce que vous mettez dans vos bouquets, moi je le disais à mes dessins" (Ibidem: 1070).
} 
rendant impossible, à l'horizon du texte, la réalisation du programme narratif manifeste - mais manifeste seulement.

Dès lors, il est clair que Balzac n'avait d'autre solution textuelle que de prendre à rebours sa propre pratique narrative dominante et les réfections successives qui ont affecté, en profondeur, la nature structurelle du Lys, conduisant à abandonner l'hypothèse d'abord privilégiée du récit enchâssé au profit de cette forme paradoxale de confession se comprennent d'elles-mêmes : Félix se devait d'être le narrateur du Lys parce que lui seul pouvait l'être, sauf à saper dangereusement tout l'édifice de la Comédie Humaine. Il fallait qu'il prît à son compte ce roman d'apprentissage avorté où se joue comme l'assomption de l'incompétence.

C'est qu'à l'insuffisance du personnage (du moi-narré), conforme, et même porteuse de dynamique textuelle dans semblable cadre générique, s'ajoute la déchéance d'un narrateur (le moi-narrant) qui se montre non seulement incapable de déchiffrer le discours d'autrui, mais jusqu'au sien propre - il fallait calculer la portée de votre discours — avec lequel Balzac se devait de combattre toute confusion sous peine d'attirer sur son grand œuvre tout entier le terrifiant soupçon de l'incompétence herméneutique. Et Balzac d'abandonner volontiers à Félix le rôle ingrat du scripteur incapable de dépasser la lettre, sans même parler de l'esprit. "Ah! si dans ces moments où je redoublais de froideur vous m'eussiez prise dans vos bras, je serais morte de bonheur. J'ai parfois désiré de vous quelque violence" (BALZAC, Le Lys:1216), est-il condamné à s'entendre dire, qui plus est doublement trop tard, incapable qu'il est de se représenter les mécanismes du refoulement ni de la censure ${ }^{14}$ et, encore moins, de dépasser les contradictions entre un corps qui réclame l'amour et un discours qui le pose comme tabou.

L'histoire de ce trop tard au carré, c'est ce qu'ont à charge de dire les textes liminaires qui balisent le parcours critique et portent condamnation de ce "je" qui demeure bloqué dans sa logique passionnelle - entendre évidemment individuelle et, partant, antisociale. Par le dispositif dialectique des deux seuils du récit, Balzac met son texte à distance de celui de Félix: mieux, du premier au second, il y a tout l'espace d'une véritable distanciation qui n'a d'autre objet que de déposséder Félix d'une parole dont il n'a pas su faire usage et que Balzac délègue à de plus compétents que lui, Natalie en

${ }^{14}$ Le comble est atteint quand Natalie lui reproche de ne pas se censurer!( Ibidem) 
l'occurrence, au terme d'une dialectique de l'adhésion et de la réfutation qui rend ostensible le protocole de lecture du texte.

L'envoi initial de Félix posait l'élucidation de soi comme achevée alors qu'elle n'était qu'erronée et fautive; la lettre d'adieu de Natalie stigmatise les obstacles de cette transparence qui se leurre, dénonce la mauvaise foi et désigne les lignes de fuite et les points aveugles de cette logique narrative et des présupposés idéologiques qui l'induisent: c'est en cela qu'elle est bien la lectrice cachée de Balzac, celle qui, idéalement, refuse de se payer de mots et vient restaurer un rapport moins lointain et moins aliéné entre les mots et choses. Sans compter qu'elle n'est pas sans contribuer puissamment au plaisir du texte, quand le lecteur - le vrai, celui-là - peut à bon compte, par son truchement, se donner le satisfecit de se reconnaître pour meilleur lecteur de l'histoire de Félix que lui-même ne l'avait été.

À replacer le destin de Félix dans le cadre élargi de la Comédie Humaine, on conçoit mieux la stratégie de progressif effacement menée à son encontre: sa prompte chute dans les oubliettes des histoires balzaciennes prononce assez sa déchéance, sur laquelle lui-même anticipe d'ailleurs, et dès Le Lys, où il s'ouvre à Natalie de ce que Charles X l'a démis de ses fonctions d'agent du crédit et du renseignement royaux (BALZAC, Le Lys:1225). A persévérer dans son essence et à persister dans des travers dénoncés ainsi qu'il le fait dans Une Fille d'Eve où il va jusqu'à épouser une... Marie-Angélique, cédant une nouvelle fois à la tentation d'un angélisme qu'il devrait pourtant tenir pour dangereuse, il ne pouvait en être autrement. La sanction narrative ne se fait pas attendre:

"Parmi les hommes remarquables qui durent leur destinée à la Restauration et que, malheureusement pour elle, elle mit avec Martignac en dehors des secrets du gouvernement, on comptait Félix de Vandenesse, déporté comme plusieurs autres à la Chambre des pairs aux derniers jours de Charles X." (BALZAC, Une fille d'Eve, 1961:840) 
Et ce sont les filles d'Eve précisément qui inquiètent jusqu'à la performance du discours de Félix ${ }^{15}$ au point que l'on est fondé à se demander si, pas davantage donc qu'il n'est un lecteur pertinent de son texte, il en est véritablement le narrateur, tant sa voix est, in fine, sinon étouffée, du moins sensiblement assourdie par le déploiement d'une polyphonie qui fait entendre les grandes orgues de deux points de vue féminins (successivement Henriette puis Natalie) qui sont donnés comme plus autorisés que le sien à lire et à dire le réel. Alors, c'est toute l'entreprise réduplicatrice de Félix qui se trouve déjouée - fourvoyée et, au propre, dévoyée qu'elle est - et sa logique de l'enfermement dénoncée pour ce qu'elle est: illusoire et inacceptable de n'être plus aucunement en prise sur l'histoire.

Il faut maintenant en revenir aux hésitations et aux repentirs balzaciens qui ont présidé aux multiples va-et-vient du Lys à l'intérieur des différentes sections de la Comédie Humaine qui pourraient bien, dans cette perspective de mise sous tutelle de voix narratives trop discordantes ou trop désaccordées pour être autorisées à se faire entendre sans de puissants correctifs, n'être pas de pure contingence. Peut-être le définitif retour à la ruralité du texte est-il à mettre au compte, plus globalement, d'une relecture nécessaire, non plus du seul Lys, mais de l'ensemble de la Comédie Humaine, jugée opportune par Balzac en 1845. Pourquoi en effet adjoindre à des romans dont la profonde cohérence ne fait pas de doute - Le Médecin de campagne, Le Curé de village ou leur envers contre-utopique en l'espèce des Paysans - un texte qui leur est aussi hétérogène que Le Lys dans la vallée? Parce qu'il participe, de façon très évidente, mais très évidemment aussi superficielle, d'une inscription campagnarde? L'explication tient trop du truisme pour être vraiment honnête.

À l'inverse, à interroger l'inscription problématique du Lys au sein de la Comédie Humaine sous le rapport de la subversion de la visée historique

is Signalons qu'encore une fois, c'est aux mécanismes de l'inversion systématiques des programmes narratifs que Félix est livré, lui qui avouait à Natalie ambitionner un tout autre programme : "Je suivis le plan que j'avais arrêté pendant ma retraite à Saché. Je me jetai dans le travail, je m'occupai de science, de littérature et de politique; j'entrai dans la diplomatie à l'avènement de Charles $X$, qui supprima l'emploi que j'occupai sous le feu Roi. Dès ce moment je résolus de ne jamais faire attention à aucune femme si belle, si spirituelle, si aimante qu'elle pût être." ( BALZAC, Le Lys : 1225). 
avouée, de la fragilisation et de la déstabilisation du sens obvie, on récupère de la rentabilité critique; il en est du retour du Lys dans les Scènes de lá vie de campagne comme du retour des Lys dans le Lys lui-même: "l'espace campagnard cess[e] d'être celui des certitudes pour s'ouvrir aux errances d'une mémoire à la dérive et, à la limite, s'y défaire" (MAHIEU, 1988).

$\mathrm{Si}$, désormais, la terre elle-même ment, il y a matière à bien des relectures...

Resumo: Seria insuficiente dizer que Le Lys dans la vallée de Balzac desfruta do estatuto de "texto sagrado", ao ponto de ser por este sufocado e imobilizado pelo terreno critico. $O$ presente artigo gostaria de dar novamente a palavra a um texto que Balzac concebia como um pivô - estético, ético, semântico - de sua grande obra. Tal objetivo nos obriga a passar o texto pelo crivo de uma abordagem plural genética, genérica, narratológica, metatextual - integrando a inscrição, várias vezes repensada, do Lys na economia geral da Comédie Humaine. Ora em todos esses niveis, é uma poética da ambigiiidade que prevalece, com uma escrita em duplo registro onde, como Penélope, o autor desfaz a obra do narrador e vice-versa. Em Balzac, aquele que pensa e aquele que escreve formam duas instâncias que se contrariam. Por detrás das hesitações, remanejamentos e arrependimentos que acompanham a história editorial do texto, a figura do intelectual critico se mostra através de uma escrita que se nega ela própria.

\section{RÉFÉRENCES BIBLIOGRAPHIQUES}

BALZAC, Honoré de. Euvres Complètes. Une fille d'Eve. Paris: Le Club français du livre, 8, 1961.

BALZAC, Honoré de. CEuvres Complètes. Le Lys dans la vallée. Paris: Pléiade, t.IX, 1978.

BALZAC, Honoré de. Le Contrat de mariage : Paris, éd. P. Barbéris, le Livre de Poche, 1984. 
BROMBERT, Victor. Natalie ou le lecteur caché de Balzac. In: Mouvements premiers. Études critiques offertes à Georges Poulet. Paris: José Corti, 1972.

DONNARD, Jean-Hervé. Notes. In: Balzac, Honoré. Le Lys dans la vallée. Paris : Gallimard, Pléiade, t.IX, 1978.

FRAPPIER-MAZUR, Lucienne. L'expression métaphorique dans la Comédie Humaine. Domaine social et psychologique. Paris : Klienksieck, 1976.

MALANDAIN, Gabrielle. Dire la passion, écrire le Lys dans la vallée. In: Romantisme, $\mathrm{n}^{\circ}$ 62, 1988, p. 31-39.

MAHIEU, Raymond. Les lapsus du Lys dans la vallée. In Balzac, Le Lys dans la vallée. Paris : Romantisme-Colloques, 1993, p. 97-105.

MAHIEU, Raymond.: Le Lys dans la vallée. Scènes de la vie de campagne : les enjeux d'une inscription problématique. Paris, $5^{\mathrm{c}}$. Colloque du GIRB, 1988.

MURA, Alice. In: Balzac, "le Lys dans la vallée". RomantismeColloques. Paris: Sedes, 1993. ROUDAUT, Jacques. Préface. Balzac, Le Lys dans la vallée. Paris: éd. Presses de la Renaissance, 1977

ROUSSET, Jean. Forme et signification. Paris: José Corti, 1962.

WEINMAN, Heinz. Bachelard et l'analyse du roman: structure des thèmes et des images dans le Lys. Revue des Sciences humaines, $\mathrm{n}^{\circ} 157,1975$, $\mathrm{p}$. 21-41. 